\title{
SPECIFICS OF IMPLEMENTING A HYBRID INTELLIGENT IMAGE GEOREFERENCING SYSTEM
}

\author{
Dmitry V. Berezkin ${ }^{1}$, Andrew V. Proletarsky ${ }^{2}$, Nadezhda A. Sukhorukova ${ }^{3}$ \\ and Ruslan A. Kamalov ${ }^{4}$ \\ ${ }^{1}$ Ph. D., associate Professor, Department of Computer systems and networks \\ ${ }^{2}$ Doctor of Technical Sciences, Professor, Dean of the Faculty of Informatics and Control Systems \\ ${ }^{3}$ master's degree student, Department of Information security \\ ${ }^{4}$ bachelor's degree student, Department of Computer systems and networks \\ Bauman Moscow State Technical University, 5c1, $2^{\text {nd }}$ Baumanskaya st., 105005, Moscow, Russian Federation
}

\begin{abstract}
The ability to determine the coordinates of objects from their photos is an urgent task and can be used in the analysis of unstructured information in social networks, to solve new problems in the field of IoT and BigData. The article develops the approach to geolocation of photos proposed by the authors in previous works and is devoted to the implementation of artificial intelligence and computer vision methods used to solve this problem. The questions of selection, design, and training of a neural network are considered, and the results of final experiments on determining the geolocation of photos are presented.
\end{abstract}

\section{KEYWORDS}

Geolocation Estimation, Scene Classification, Hybrid Artificial Intelligence, Computer Vision, Digital Image Processing, Neural Network, Deep Learning

\section{INTRODUCTION}

Determining the location of an object based on its photo is necessary for solving a number of tasks. This is an example of detecting and filtering fake news. In this case, a large amount of data coming from various sources is subject to analysis.

Although many cameras and smartphones allow you to store location information as a part of image metadata, it is easy to change or delete such data. So users of mobile devices can disable geolocation for privacy reasons or to reduce the load on the battery. When posting images on various Internet resources, such as social network Facebook, Twitter or Instagram, the photo metadata is automatically removed during loading. Because of this, it is often impossible to determine the location from the image.

Artificial intelligence technologies can help solve the problem of automatic geo-linking of images. With their help, it becomes possible to determine the location of a photo shoot by recognizing various informative objects on it, such as signs, state license plates, information and advertising signs, attractions, as well as architectural features and some other features, which allows you to solve the actual problem of creating intelligent technologies for georeferencing images that do not have geolocation tags. These technologies should allow you to replace the human operator when solving problems of determining the point of taking a photo. In the previous work of the authors (Dmitry Berezkin, Andrew Proletarsky, Valentin Sidorov, Ilya Sergeev, 2019), the authors developed an approach to solving this problem by processing and analyzing images using hybrid artificial intelligence methods. At this stage of research, the main methods of image mining were implemented, which are necessary for predicting the coordinates of objects from their images. 


\section{RELATED WORKS}

Currently, a number of companies are working on solving the problem of georeferencing images. For example, Google announced that its search engine will soon be able to determine the place where a particular photo was taken. To do this, the company's employees created a neural network called PlaNet (Weyand T., Kostrikov I., Philbin J., 2016), in the database of which more than 90 million images were uploaded indicating the places where they were taken. When performing a location search, PlaNet will compare the image with images from the database at the pixel level. If it matches, it will give you an idea of where the photo was taken. The basic principles of recognition are described in the article (Weyand T., Kostrikov I., Philbin J., 2016). It should be noted that the system is still far from ideal. During the first tests, the program was able to correctly identify the country only in $28.4 \%$ of cases and the continent in $48 \%$.

The paper (Nam N. Vo, Nathan Jacobs, James Hays, 2017) presents the Im2GPS project, in which the geo-location estimation is based on comparing the original photo with 6 million prepared images from Flickr, for which GPS coordinates are set. Comparative results of the geo-location assessment of the PlaNet and Im2GPS projects are described in (Nam N. Vo, Nathan Jacobs, James Hays, 2017; Eric Müller-Budack Kader Pustu-Iren Ralph Ewerth, 2018). These results also indicate that the accuracy of geolocation of objects from photos is not yet high.

\section{GEOLOCATION OF PHOTOS - TECHNIQUES AND METHODS}

Recognizing the language in which the text is written on the image is important for determining the location from the photo. This allows you to reduce the search to a group of countries if the recognized language is the official language for several countries, or to identify a specific country if the language is unique.

The search and determination of the language belonging in the image takes place in several stages. The first step is to search for text in the image. Computer vision methods can be used to implement this function.

To improve the performance of the text search and extraction subsystem, you need to perform a number of actions with pre-prepared images. Enlarging the image without losing its quality will allow you to better search for text. You also need to split the image into segments to exclude areas that can't exactly contain text. To do this, use the OpenCV library boundary search functions. The program modules are implemented in the C\# programming language using the OpenCV computer vision library and the Tessnet text library.

After the image is processed by this detector, all areas containing text information will be highlighted on the image, and the coordinates of the location of these areas will be known. The second step is to extract the text from the image. The open source software Tesseract is used for this purpose (Thakare, S., Kamble, A., Thengne, V., Kamble, U. R., 2018).

In order to automatically determine which language the text is written in, the image language detection module uses the open Langdetect library written for Python or Java. This library generates language profiles based on xml annotations from Wikipedia, and determines the language of the text using Bayesian filtering. Its accuracy is $99 \%$ for 53 languages.

The analysis of the language recognition of the text in the photo showed that if the text in the photo is taken large, then the recognition is high-quality. When there are multiple languages in the photo, characters that are included in multiple languages are selected. This option is very difficult to recognize and does not allow you to determine the language for short texts when there is not a single whole word in the image. If the text was captured from a long distance, the resolution after image segmentation will not be enough to find the text in the image. This circumstance may become an objective restriction on the use of text labels on the image to solve the problem of geolocation.

It was decided to use neural networks to classify objects and get the most accurate coordinates from photos. This is justified by the fact that they have a flexible algorithm that allows you to smooth out the errors of the training sample and get a high speed of information search compared to the search for key points. This is due to the fact that photos usually show several complex objects and extracting key points can take a very long time.

Multi-layer convolutional neural networks can be used to solve the problem of object classification in an image (Rusnachenko N.L., Loukachevitch N.V., 2018; Fedorenko Y. S., Gapanyuk Y. E., 2018; Daria A. Eroshenkova et al., 2020; Weyand T., Kostrikov I., Philbin J., 2016; Neha Sharma, Vibhor Jain, Anju 
Mishra, 2018; Mingyuan Xin, Yong Wang, 2019). At the same time, training and tuning of the neural network parameters should be performed taking into account the specifics of the arrays of processed photos.

As a result of the analysis of various implementation options for the neural network, The Residual neural network (ResNet) was chosen as its architecture, which. As it showed better results compared to the convolutional neural network when increasing the network depth due to better opportunities for its optimization (He, Kaiming; Zhang, Xiangyu; Ren, Shaoqing; Sun, Jian 2016).

It should also be noted that there are a large number of image data sets for training the ResNet neural network to solve the problem of their classification (He, Kaiming; Zhang, Xiangyu; Ren, Shaoqing; Sun, Jian 2016). The images include sets of characteristics that are specified manually by experts. These features usually do not include the geographical coordinates of images. This circumstance required developing and implementing an algorithm for creating your own training sample.

To train the neural network, we use photos with known geolocation, the number of which was limited. Adding additional photos without metadata to the training sample and determining their geolocation allows you to expand the amount of data for subsequent analysis and improve the quality of neural network training.

To create a training sample, a subsystem for preparing large arrays of images is designed and the algorithm for searching for similar photos was developed. For each photo from the original selection, similar photos are searched using the Bing Image Search service. First, a search query is created and the original photo is sent to it. The result is received in JSON format, after parsing it, the program determines the url of the link to similar photos. The resulting photos are then saved and the metadata file is updated with the geotags of the new photos.

As a result of executing this module, the original sample is increased by almost 100 times. All data is stored on the remote file storage. This creates a hierarchy of directories where data is uploaded. Uploading photos to remote storage updates the metadata file with links to photos.

The neural network was trained on 17885 photos, and the original dataset was divided into batches of 512 objects. The neural network showed the maximum efficiency of training in photos with a dimension of 256 by 256 pixels. The training was conducted on $64 \mathrm{CPU}$ cores. The network with 34 and 50 layers slightly increased its recognition quality after 4-6 epochs, while the neural network with 152 layers had the highest quality. The quality metric was used by accuracy. The best learning rate was between 0.01 and 0.05 . On average, one epoch was trained in 4 hours.

\section{RESULTS AND DISCUSSION}

The authors implemented a neural network in the Python programming language using the PyTorch and FastAI libraries. This network was trained and final experiments were conducted to determine the geolocation of photos on test samples. The prediction results, show that the developed neural network allows recognizing objects in photos taken in different lighting conditions and in the presence of distortions.

A study of the accuracy of predicting the geographical coordinates of images was performed, which showed a high accuracy of $90 \%$ when analyzing images with a dimension of $128 \times 128$ with a small number of image classes. We studied 3 classes of geographical objects with 300-500 images in each class, and used 34 layers of the ResNet34 architecture. According to the authors' estimates, when improving the training sample by removing photos that do not belong to the desired class, the prediction accuracy can reach $99.9 \%$.

As the number of classes increased, the accuracy decreased and did not exceed 61\% (for 240 image classes). Increasing the resolution of photos and the number of layers of the ResNet neural network did not lead to an increase in accuracy, but significantly increased the time for its training (up to 12-36 hours per epoch). Analysis of this situation has shown that the training sample includes a large number of photos with incorrect coordinates when it is automatically expanded. For example, of the 23000 images analyzed, about $33 \%$ of the outliers were recorded. This indicates that the accuracy of the algorithm can be increased: to do this, you need to filter the training data and remove erroneous photos, or improve the algorithm for expanding the training sample, described in point 3. 


\section{CONCLUSION}

Experiments have shown that it is possible to determine the geolocation of images in the presence of famous sights on them. Although the accuracy achieved so far is not high and is approximately $60 \%$, it is possible to improve it by improving the quality of training sample formation.

For geolocate images that do not contain landmarks, you need to use additional informative objects, such as road signs, flags, advertising, license plates, architectural elements, road signs and traffic management features, characteristic plants, etc. For their recognition, various neural networks can be used, which are combined and combining into a hybrid neural network. At the same time, the ability to recognize such objects will allow you to interpret the results of the neural network.

\section{REFERENCES}

Daria A. Eroshenkova,Valeri I. Terekhov, Dmitry R. Khusnetdinov, Sergey I. Chumachenko, 2020. Automated Determination of Forest-Vegetation Characteristics with the Use of a Neural Network of Deep Learning. Advances in Neural Computation, Machine Learning, and Cognitive Research III. NEUROINFORMATICS 2019. Studies in Computational Intelligence, vol 856. Springer, Cham. https://doi.org/10.1007/978-3-030-30425-6_34

Dmitry Berezkin, Andrew Proletarsky, Valentin Sidorov, Ilya. Sergeev, 2019. Application of the methods of hybrid artificial intelligence to determine the location of objects by their photography. Proceedings of the $16^{\text {th }}$ International Conference on Applied Computing 2019. Cagliary, Italy, pp. 245-250.

Eric Müller-Budack Kader Pustu-Iren Ralph Ewerth, 2018. Geolocation Estimation of Photos Using a Hierarchical Model and Scene Classification. Computer Vision - ECCV 2018, pp. 575-592.

Fedorenko Y. S., Gapanyuk Y. E., 2018. The Neural Network with Automatic Feature Selection for Solving Problems with Categorical Variables. International Conference on Neuroinformatics. - Springer, Cham, 2, pp. 129-135.

He Kaiming, Zhang Xiangyu, Ren,Shaoqing, Sun Jian, 2016. Deep Residual Learning for Image Recognition. Proc. Computer Vision and Pattern Recognition (CVPR), IEEE. Retrieved 2020-04-23. // https://arxiv.org/pdf/1512.03385.pdf

Mingyuan Xin, Yong Wang, 2019. Research on image classification model based on deep convolution neural network. EURASIP Journal on Image and Video Processing, 2019, Article number 40 (2019).

Nam N. Vo, Nathan Jacobs, James Hays, 2017. Revisiting IM2GPS in the Deep Learning Era. ICCV 2017, pp 2640-2649.

Neha Sharma, Vibhor Jain, Anju Mishra, 2018. An Analysis Of Convolutional Neural Networks For Image Classification Proceedings of the International Conference on Computational Intelligence and Data Science, Volume 132, 2018, Pages 377-384.

Rusnachenko N.L., Loukachevitch N.V., 2018. Extracting sentiment attitudes from analytical texts via piecewise convolutional neural network. Proceedings of 20 International Conference Damdid/RCDL-2018, pp. 186-192.

Thakare S., Kamble A., Thengne V., Kamble U.R., 2018. Document Segmentation and Language Translation Using Tesseract-OCR. 2018 13th International Conference on Industrial and Information Systems, ICIIS 2018 - Proceedings, pp. 148-151.

Weyand T., Kostrikov I., Philbin J., 2016. Planet-photo geolocation with convolutional neural networks. European Conference on Computer Vision. - Springer, Cham, pp. 37-55. 